\title{
Pediatric Cirrhotic Variceal Bleed Precipitating Diabetic Ketoacidosis
}

\author{
Rachel E. Bridwell ${ }^{1}$, Neil P. Larson ${ }^{1}$, Jesse Wray ${ }^{1}$, Amber Cibrario ${ }^{1}$, Joshua Oliver ${ }^{1}$ \\ 1. Emergency Medicine, Brooke Army Medical Center, Fort Sam Houston, USA
}

Corresponding author: Rachel E. Bridwell, r.e.bridwell@gmail.com

\begin{abstract}
Cirrhosis and its associated complications such, as variceal bleeding, are rare in children, carrying significant morbidity and mortality. Leading causes of cirrhosis in the pediatric population include infection, neoplasm, and metabolic and genetic disorders, which is in contrast to the adult population. Acute gastrointestinal bleeding, as seen with variceal bleeding, has been previously associated with diabetic ketoacidosis through a multifactorial relationship. The case was complicated by hypovolemic shock whose resuscitation and subsequent transfusion was associated with cardiac overload. We highlight the need for balanced, judicious resuscitation in these individuals as well as the need for heightened awareness of coexisting pathologies such as diabetic ketoacidosis.
\end{abstract}

Categories: Emergency Medicine, Pediatrics, Gastroenterology

Keywords: pediatric cirrhosis, variceal bleeding, diabetic ketacidosis

\section{Introduction}

Ruptured gastroesophageal varices are a medical emergency and the most frequent source of gastrointestinal bleeding in pediatric patients with liver cirrhosis [1]. While a broad differential diagnosis exists for the etiology of underlying pediatric liver cirrhosis, a recent study cited Wilson's disease, biliary atresia and cryptogenic sources as the most frequent culprits for patients ages 18 years of age and younger [2]. However, recent trends demonstrate an increased incidence of non-alcoholic steatohepatitis, largely reflecting the worldwide obesity problem [3,4]. Bleeding esophageal varices complicate approximately $16 \%$ of affected patients, carrying a 19\% 30-day mortality [2]. There is a multifactorial relationship between acute gastrointestinal bleeding (AGIB) and diabetic ketoacidosis (DKA). Though pediatric data is limited, adult studies suggest that in cirrhotic patients hospitalized for DKA, higher presenting serum glucose levels are associated with increased rates of AGIB, and increased mortality may exist for patients experiencing AGIB compared with those who do not [5].

\section{Case Presentation}

A 17-year-old $112 \mathrm{~kg}$ male with a history of Acute Lymphoblastic Leukemia (ALL) in remission and Type 2 Diabetes Mellitus was brought in by ambulance to a pediatric emergency department for altered mental status. He was found unconscious in a gas station restroom with hematemesis and rectal bleeding. His mother denied a history of coagulopathy or liver dysfunction. Initial vital signs were a heart rate of 125 beats

per minute, a blood pressure of $144 / 52 \mathrm{~mm} \mathrm{Hg}$, temperature of $97.9^{0} \mathrm{~F}$ oral, a respiratory rate of 30 breaths per minute, with an oxygen saturation of $100 \%$ on 15 liters by non-rebreather mask. Physical exam was notable for mildly depressed level of consciousness though rousable, diffuse abdominal pain, and blood per rectum. Left distal femoral intraosseous access and 20 gauge intravenous forearm access were obtained en route. Initial point of care venous blood gas demonstrated a $\mathrm{pH}$ of 7.18, $\mathrm{pCO}^{2}$ of $49.3 \mathrm{~mm} \mathrm{Hg}$, bicarbonate of $18 \mathrm{mmol} / \mathrm{L}$, glucose of $691 \mathrm{mg} / \mathrm{dL}$ and an anion gap of $21.4 \mathrm{mmol} / \mathrm{L}$. Relevant initial lab results included a white blood cell count of 27,600 cells/mm, hemoglobin of $9.1 \mathrm{~g} / \mathrm{dL}$, platelets of 9,000/uL, fibrinogen of 188 $\mathrm{mg} / \mathrm{dL}$ (normal 200-450 mg/dL), and a D-dimer of $2.19 \mathrm{ug} / \mathrm{mL}$ (normal $0.05 \mathrm{ug} / \mathrm{mL}$ ). An insulin infusion was initiated at $0.1 \mathrm{units} / \mathrm{kg} / \mathrm{hr}$ and one unit of packed red blood cells (pRBCs) was transfused. Due to hypotension with a repeat blood pressure of of $64 / 42$, an epinephrine infusion was initiated at 0.05 $\mathrm{mcg} / \mathrm{kg} / \mathrm{min}$ for hypotension. After a large volume of hematemesis, the patient had a further decrease in mental status and was intubated followed by nasogastric tube placement. Simultaneously, three units pRBCs, two units fresh frozen plasma (FFP), 5 units of cryoprecipitate, 1 six-pack of platelets, and $7 \mathrm{~g}$ of factor VII replacement were transfused and the patient was admitted to the pediatric intensive care unit (PICU) for urgent upper endoscopy by gastroenterology. On arrival in the PICU, the patient had another large bloody bowel movement with subsequent hypotension, requiring additional transfusion of two units pRBC and one unit FFP. Upper endoscopy revealed large esophageal varices with a clot in place though no further intervention was required. Emergent computed tomography revealed cirrhosis with portal hypertension. The patient's ICU course was complicated by transfusion associated circulatory overload (TACO). However, he rapidly improved and was extubated on hospital day 3 and discharged on hospital day 8.

\section{Discussion}


As compared to the adult population, pediatric liver dysfunction and cirrhosis occur more often secondary to infection, neoplasm, metabolic, and genetic disorders [6]. Though less common in the pediatric population, cirrhosis incurs rapid morbidity and mortality of $19 \%$ in the first 30-days following a variceal bleed [2]. In pediatric cirrhotic patients, variceal bleed is the most common cause of AGIB [7]. Decompensation from variceal bleeding, hepatic encephalopathy, and secondary bacterial infection all dramatically increase mortality in this population $[8,9]$.

This case highlights an unusual presentation of initial identification of cirrhosis with variceal AGIB likely precipitating DKA. While DKA in a pediatric patient usually favors a hypercoagulable state, decompensated cirrhosis likely led to the coagulopathy seen in this patient; while AGIB is seen in nine percent of adult cases of DKA, very few cases of AGIB precipitating DKA in children have been reported [10]. In a retrospective case series of 25 pediatric patients with DKA associated with AGIB, one third demonstrated, esophageal erosions, Mallory-Weiss tear, or esophagitis on endoscopy [10,11]. However, there are no reported cases of DKA secondary to variceal cirrhotic bleeding. This patient had no diagnosed history of cirrhosis and thus massive AGIB was the presenting symptom of his cirrhosis. This highlights the critical and unusual nature of this case.

Pediatric variceal bleeding secondary to cirrhosis should be aggressively resuscitated using balanced transfusion of blood products as well as early prophylaxis for spontaneous bacterial peritonitis. Investigation of both concomitant coagulopathy as well as any resulting metabolic derangements should be expeditiously pursued. The patient's severe coagulopathy again highlights this necessity.

Thromboelastography guided resuscitation has demonstrated utility in the adult cirrhotic population, achieving better hemostasis with fewer blood products used [12,13]. A balanced, judicious resuscitation mitigates further complication to include TACO, as seen in this case [13]. Endoscopic variceal ligation and sclerotherapy are both used in definitive treatment of bleeding variceal in pediatric patients; ligation has been shown to reduce rates of rebleeding as compared to sclerotherapy, though the selection of therapy is multifactorial [14]. Due to the critical nature of their presentation, these patients should be admitted to the PICU with pediatric gastroenterology available for urgent endoscopy [14].

\section{Conclusions}

Cirrhosis complicated by variceal bleeding is rare in pediatric populations but carries increased morbidity and mortality. Variceal bleeding in pediatric patients requires a balanced resuscitation with blood products and admission to a PICU with pediatric endoscopy capabilities. Emergency providers should search for concomitant derangements to include DKA in this population. To our knowledge, this is the first case where the index presentation of cirrhosis with variceal bleeding caused DKA. While rare, emergency providers should consider younger presentations of cirrhosis in comorbid patients and resuscitate aggressively but judiciously.

\section{Additional Information \\ Disclosures}

Human subjects: Consent was obtained by all participants in this study. Conflicts of interest: In compliance with the ICMJE uniform disclosure form, all authors declare the following: Payment/services info: All authors have declared that no financial support was received from any organization for the submitted work. Financial relationships: All authors have declared that they have no financial relationships at present or within the previous three years with any organizations that might have an interest in the submitted work. Other relationships: All authors have declared that there are no other relationships or activities that could appear to have influenced the submitted work.

\section{Acknowledgements}

The opinions reflected in this manuscript are those of the authors alone and do not reflect those of the United States Air Force, United States Army, Department of Defense, or Federal Government. All authors equally contributed in the creation of this manuscript, have no financial disclosures, and did not receive any external funding.

\section{References}

1. Pinto RB, Schneider ACR, da Silveira TR: Cirrhosis in children and adolescents: an overview . World J Hepatol. 2015, 7:392-405. 10.4254/wjh.v7.i3.392

2. Dehghani SM, Imanieh MH, Haghighat M, Malekpour A, Falizkar Z: Etiology and complications of liver cirrhosis in children: report of a single center from southern Iran.. Middle East J Dig Dis. 2013, 5:41-46.

3. Pinzani M, Rosselli M, Zuckermann M: Liver cirrhosis. Best Pract Res Clin Gastroenterol. 2011, 25:281-290. 10.1016/j.bpg.2011.02.009

4. Nobili V, Svegliati-Baroni G, Alisi A, Miele L, Valenti L, Vajro P: A 360-degree overview of paediatric NAFLD: recent insights. J Hepatol. 2013, 58:1218-1229. 10.1016/j.jhep.2012.12.003

5. Badipatla KR, Jadhav P, Vaddigiri S, et al.: Predictors of acute gastrointestinal bleeding in diabetic ketoacidosis: a retrospective observational study in minority population. Gastroenterol Rep. 2017, 5:293297. 10.1093/gastro/gox006 


\section{Cureus}

6. Khan S, Aqeel S, Arif M, Aziz S: Unusual presentation of a young child with cirrhosis: a case report . Ann Pediatr Child Health. 2015, 3:1064.

7. Shneider BL, Bosch J, De Franchis R, et al.: Portal hypertension in children: expert pediatric opinion on the report of the Baveno v consensus workshop on methodology of diagnosis and therapy in portal hypertension.. Pediatr Transplant. 2012, 16:426-437. 10.1111/j.1399-3046.2012.01652.x

8. Zipprich A, Garcia-Tsao G, Rogowski S, Fleig WE, Seufferlein T, Dollinger MM: Prognostic indicators of survival in patients with compensated and decompensated cirrhosis. Liver Int. 2012, 32:1407-1414. 10.1111/j.1478-3231.2012.02830.x

9. Arvaniti V, D’Amico G, Fede G, et al.: Infections in patients with cirrhosis increase mortality four-fold and should be used in determining prognosis. Gastroenterology. 2010, 139:1246-1256. 10.1053/j.gastro.2010.06.019

10. Bialo SR: Rare complications of pediatric diabetic ketoacidosis. World J Diabetes. 2015, 6:167-174. 10.4239/wjd.v6.i1.167

11. Faigel DO, Metz DC: Prevalence, etiology, and prognostic significance of upper gastrointestinal hemorrhage in diabetic ketoacidosis. Dig Dis Sci. 1996, 41:1-8. 10.1007/BF02208576

12. De Pietri L, Bianchini M, Montalti R, et al.: Thrombelastography-guided blood product use before invasive procedures in cirrhosis with severe coagulopathy: a randomized, controlled trial. Hepatology. 2016, 63:566573. 10.1002/hep.28148

13. Rout G, Shalimar, Gunjan D, et al.: Thromboelastography-guided blood product transfusion in cirrhosis patients with variceal bleeding: a randomized controlled trial. J Clin Gastroenterol. 2020, 54:255-262. 10.1097/MCG.0000000000001214

14. Zargar SA, Javid G, Khan BA, et al.: Endoscopic ligation compared with sclerotherapy for bleeding esophageal varices in children with extrahepatic portal venous obstruction. Hepatology. 2002, 36:666-672. 10.1053/jhep.2002.35278 